\title{
Vaginose Bacteriana e DNA de Papilomavírus Humano de Alto Risco Oncogênico em Mulheres Submetidas a Conização com Alça Diatérmica para Tratamento de Neoplasia Intra-epitelial Cervical de Alto Grau
}

Bacterial Vaginosis and High-risk HPV-DNA in Women Submitted to Diathermic Conization for the Treatment of High-grade Cervical Intra-epithelial Neoplasia

\author{
Michelle Garcia Discacciati ${ }^{1}$, Silvia Helena Rabelo-Santos ${ }^{1}$, Elisabete Aparecida Campos ${ }^{1}$, \\ José Antonio Simões², Sophie Françoise Mauricette Derchain², \\ Luís Otávio Zanatta Sarian², Luiz Carlos Zeferino ${ }^{2}$
}

\section{RESUM0}

Objetivo: avaliar a associação entre vaginose bacteriana (VB), DNA de papilomavírus humano (HPV) de alto risco e anormalidades citopatológicas cervicais em mulheres submetidas a conização diatérmica devido a neoplasia intra-epitelial cervical de alto grau (NIC 2 ou 3). Métodos: estudo clínico descritivo, no qual foram incluidas 81 mulheres submetidas a conização diatérmica devido a NIC 2 ou 3. A citologia (CO) inicial foi colhida na época da realização da biópsia dos casos suspeitos e também foi utilizada para verificar a presença de $V B$. Antes da conização diatérmica foi coletado material para a detecção de DNA de HPV de alto risco, por meio da captura de híbridos II (CH II). Após a conização diatérmica foi agendado retorno em 4 meses, no qual eram realizadas novas coletas de $\mathrm{CO}$ e de $\mathrm{CH}$ II. Vinte e sete mulheres apresentaram VB e 54 não apresentaram esta alteração. A análise estatística foi realizada por meio do cálculo dos odds ratios (OR) para as relações entre a detecção do HPV e a presença de anormalidades citológicas com a presença de VB, antes e após a conização, considerando-se intervalos de confiança de 95\% (IC 95\%).

Resultados: a detecção de DNA de HPV de alto risco antes da conização foi semelhante nos dois grupos (89\%). Após a conização, esta detecção foi igual a 26 e 18\%, respectivamente, nos grupos com e sem VB (OR=1,5 IC 95\% 0,5 a 4,6). Ainda após a conização, 41\% das pacientes com VB e 20\% das sem VB apresentaram anormalidades citológicas $(O R=2,7$; IC 95\% 1,0 a 7,4). Analisando-se exclusivamente as 22 mulheres com anormalidades citológicas em seus exames realizados aproximadamente quatro meses após a conização diatérmica, 83\% daquelas com VB também apresentaram testes positivos para DNA de HPV, comparadas a 50\% daquelas sem VB (OR=5,0; IC 95\% 0,5 a 52,9).

Conclusão: mulheres com VB apresentaram maior proporção de anormalidades citopatológicas depois da conização em relação às mulheres sem $V B$, ainda que estatisticamente não significativa. Esta associação não foi relacionada à presença do DNA de HPV de alto risco.

PALAVRAS-CHAVE: Vaginose bacteriana. Papilomavírus. Neoplasia intra-epitelial cervical. Colo: lesões pré-neoplásicas. Conização.

${ }^{1}$ Centro de Atenção Integral a Saúde da Mulher (CAISM), Campinas-SP;

${ }^{2}$ Departamento de Tocoginecologia, Faculdade de Ciências Médicas, Universidade Estadual de Campinas (UNICAMP). Correspondência:

José Antonio Simões

Caixa Postal 6181

Cidade Universitária Zeferino Vaz

13.083-970 - Campinas - SP

Telefones: (19) 3289-2856/ 3788 9306/Fax: (19) 32892440 e-mail: jsimoes@caism.unicamp.br

Projeto financiado parcialmente pela FAPESP processo $\mathrm{n}^{\circ}$ 00/11264-0.e CNPq processo 300354/01

\section{Introdução}

A conização do colo uterino é o procedimento indicado para o tratamento da neoplasia intraepitelial cervical (NIC) de alto graul. Entretanto, tem sido relatada a detecção de doença residual, tanto do ponto de vista histopatológico como detecção de DNA de HPV, em uma proporção considerável dos casos, o que aumenta a possibilidade de evolução para a doença invasiva ${ }^{2-5}$. 
Vários fatores têm sido considerados indicadores para a doença residual, tais como a idade, a gravidade da lesão, o comprometimento das margens e a paridade; porém, a presença de infecção por HPV após a conização tem sido o fator indicativo encontrado com maior freqüência ${ }^{6-12}$.

Alterações de flora vaginal sugestivas de vaginose bacteriana (VB) ocorrem com freqüência significativamente maior entre as mulheres com anormalidades citológicas cervicais em comparação com aquelas cuja citologia cervical é normal1 $1^{3,14}$. Há, também, associação significativa entre DNA de HPV e flora indicativa de VB ${ }^{15}$. Assim, vem sendo sugerido que a VB também poderia ter papel importante no desenvolvimento da NIC devido às nitrosaminas oncogênicas produzidas pelas bactérias anaeróbicas e, ainda, devido ao estímulo para a produção de citocinas, como a interleucina 1 beta $1^{6,17}$.

O objetivo deste estudo foi avaliar as alterações da flora vaginal sugestivas de VB e a detecção de DNA de HPV de alto risco oncogênico, antes e após a conização diatérmica para tratamento de NIC de alto grau (NIC 2 ou NIC 3), a fim de correlacioná-los com a recorrência de anormalidades citológicas (atipias de células escamosas de significado indeterminado - ASCUS, NIC e atipias de células glandulares - ACG).

\section{Pacientes e Métodos}

Os dados foram obtidos de um amplo estudo clínico prospectivo realizado anteriormente no Centro de Atenção Integral a Saúde da Mulher (CAISM) e aprovado pelo Comitê de Ética da Faculdade de Ciências Médicas da UNICAMP. No presente estudo, foram incluídas 81 mulheres que tiveram indicação para conização diatérmica do colo uterino devido ao diagnóstico histológico de NIC 2 ou 3.

Todas as pacientes tinham exames citológico (CO), colposcópico ou histológico iniciais sugestivos de NIC. Na primeira consulta e após a assinatura do Termo de Consentimento Livre e Esclarecido, estas mulheres foram submetidas a novo exame ginecológico, no qual foram realizados: nova coleta de $\mathrm{CO}$, exame colposcópico com ácido acético a 3\% e biópsia de áreas morfologicamente sugestivas de câncer.

A conização por alça diatérmica foi indicada para todas as mulheres com CO sugestiva de NIC 3 ou com resultado histológico pela biópsia de NIC 2 ou 3, e foi realizada durante a primeira fase do ciclo menstrual. Pacientes cuja biópsias mostra- ram câncer foram devidamente tratadas, não sendo incluídas no estudo.

Antes do início da conização diatérmica foi coletado material para a detecção de DNA de HPV de alto risco, por meio da captura de híbridos II (CH II). Após a conização diatérmica, foi agendado retorno para quatro meses. Nessa consulta de controle, foram realizadas: nova coleta de $\mathrm{CO}$, colposcopia e colheita de material para $\mathrm{CH}$ II.

Os resultados de $\mathrm{CH}$ II foram considerados positivos quando a carga viral era maior do que uma unidade relativa de luz (URL), o que corresponde a $1 \mathrm{pg} / \mathrm{mL}$ de DNA de HPV, equivalente a 0,1 cópia de vírus/célula. Os esfregaços cervicais foram corados pelo método de Papanicolaou e avaliados no Laboratório de Citopatologia do CAISM/UNICAMP, quanto à presença de alteração de flora vaginal sugestiva de VB segundo os critérios de Schnadig et al. ${ }^{18}$ (em especial pela presença de clue cells) e quanto à presença de anormalidades citológicas (ASCUS, NIC 1, NIC 2, NIC 3 e ACG). Foram comparados os resultados antes da conização e cerca de quatro meses após a sua realização.

Vinte e sete pacientes apresentaram esfregaço sugestivo de VB antes da conização e 54 não apresentaram esta alteração.

A análise estatística foi realizada com o cálculo dos odds ratios (OR) para as relações entre a detecção do HPV e a presença de anormalidades citológicas com a presença de VB, antes e após a conização, considerando-se intervalos de confiança de 95\% (IC 95\%).

\section{Resultados}

A proporção de mulheres com DNA de HPV detectável, antes da conização, foi a mesma nos grupos estudados quanto à presença de VB, atingindo 89\% em ambos (OR=1,0; IC 95\% 0,2 a 4,3). Aproximadamente quatro meses após a conização diatérmica, a proporção de mulheres com DNA de HPV detectável foi reduzida para $26 \%$ entre aquelas com VB e, entre as que não tinham VB, para 18\% (OR=1,5; IC 95\% 0,5 a 4,6). Já a presença de anormalidades citológicas, na avaliação realizada quatro meses após a conização diatérmica, foi de $41 \%(11 / 27)$ entre as mulheres com VB, contrastando com 20\% $(11 / 54)$ entre aquelas sem VB (OR=2,7; IC95\% 1,0 a 7,4) (Tabela 1).

A freqüência de comprometimento das margens endocervicais foi similar nos dois grupos de mulheres submetidas à conização diatérmica, 30\% nas pacientes com VB e $22 \%$ nas pacientes sem VB. 
Analisando-se exclusivamente as 22 mulheres com anormalidades citológicas em seus exames realizados aproximadamente quatro meses após a conização diatérmica, $83 \%$ daquelas com VB também apresentaram testes positivos para DNA de HPV, comparadas a 50\% daquelas sem VB (OR=5,0; IC 95\% 0,5 a 52,9) (Tabela 2).

Tabela 1 - Detecção do DNA de HPV e anormalidades citológicas em função da presença de vaginose bacteriana antes da conização diatérmica.

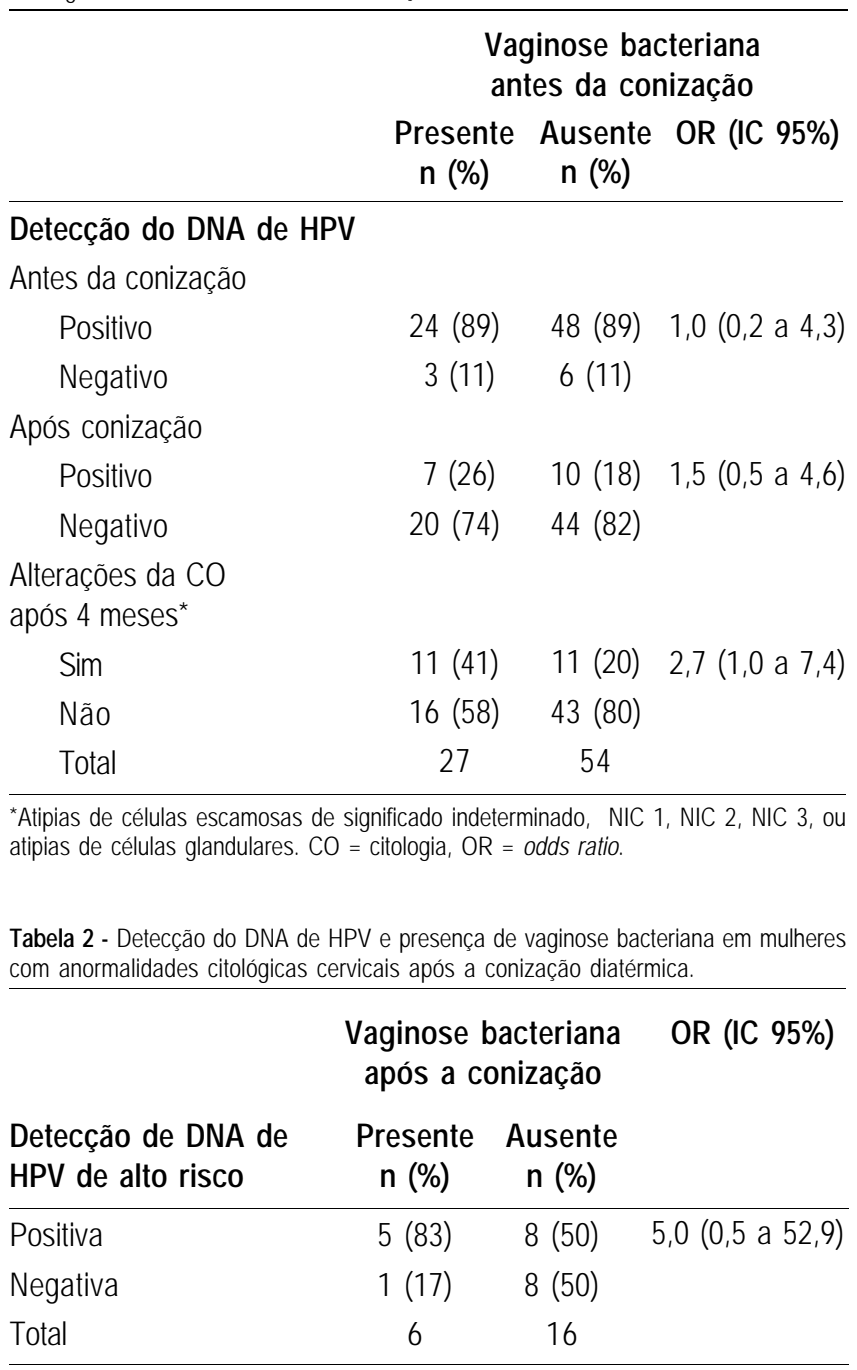

$\mathrm{OR}=$ odds ratio

\section{Discussão}

Foi observado neste estudo que não houve diferença na freqüência de detecção de DNA de HPV de alto risco entre os grupos de pacientes com ou sem VB, antes da conização diatérmica. Nos dois grupos houve diminuição da freqüência de detecção de DNA de HPV aproximadamente quatro meses após a conização. Estudos anteriores também demonstraram diminuição significativa da detecção de DNA de HPV após tratamento da NIC por conização diatérmica, o que sugere que este procedimento contribui para o desaparecimento do vírus ${ }^{2,9,19}$.

A freqüência de anormalidades citológicas cervicais após a conização foi maior nas pacientes com VB presente antes da conização em comparação com as mulheres sem VB. Estes dados, embora não tenham sido estatisticamente significativos, estão em acordo com outros estudos que demonstraram associação positiva entre anormalidades citológicas cervicais e alterações de flora vaginal sugestivas de $\mathrm{VB}^{13,20,2} 1$. Cumpre ressaltar que um dos principais fatores considerados como sendo de risco para a ocorrência de doença residual após conização diatérmica, ou seja, o comprometimento das margens, foi similar em ambos os grupos aqui estudados.

Considerando-se apenas as 22 pacientes de ambos os grupos que apresentaram anormalidades cervicais após a conização, este estudo demonstrou que naquelas pacientes que apresentaram VB após a conização a freqüência de DNA de HPV foi maior do que a freqüência encontrada nas pacientes que não apresentaram VB após a conização. Esses achados são semelhantes aos encontrados por alguns pesquisadores que, analisando a flora vaginal por meio de métodos bacterioscópicos padronizados, também concluíram que a mudança da flora normal para uma flora anaeróbica sugestiva de VB está associada a maior freqüência na detecção de DNA de HPV ${ }^{15,22}$.

Todavia, ainda não está completamente esclarecido se as bactérias anaeróbicas envolvidas na VB e seus produtos metabólicos poderiam ter efeito direto na evolução das neoplasias do colo uterino.

Apesar das restrições, os achados deste trabalho justificam a realização de estudos futuros, com maior número de casos para esclarecer o verdadeiro papel da VB na persistência de NIC após a conização e também se a VB seria um fator de risco ou apenas um distúrbio de flora associado às NIC e à infecção pelo HPV.

\section{Abstract}

Purpose: to analyze the association between bacterial vaginosis $(B V)$, high-risk HPV DNA, and Pap smear abnormalities in women submitted to diathermic conization for the treatment of high-grade cervical intraepithelial neoplasia (CIN 2 or 3 ). 
Methods: a descriptive clinical study with 81 women submitted to diathermic conization for the treatment of CIN 2 or 3. Initial Pap smear was performed by the time of the biopsy and was also used to verify the presence of BV. Prior to conization, samples for the detection of high-risk HPV DNA through hybrid capture II (HC II) were collected. A control visit was scheduled for four months after the conization to repeat these tests. Twenty-seven women were found to have $B V$ and 54 were not. Statistical analysis comprised odds ratios (OR) to assess the correlations between $B V$ and HPV detection before and after diathermic conization and cytological abnormalities. All analyses were performed with a $95 \%$ confidence interval (95\% CI).

Results: high-risk HPV DNA detection before conization was identical in both groups (89\%). After conization, HPV DNA detection decreased to 26 and $18 \%$ in the groups with and without $B V$, respectively $(O R=1.5 ; 95 \%$ CI 0.5 to 4.6$)$. In addition, $41 \%$ of the women with $B V$ and $20 \%$ without $B V$ showed Pap smear abnormalities (OR=2.7; 95\% CI 1.0 to 7.4). Regarding these 22 women with Pap smear abnormalities approximately four months after the diathermic conization, $83 \%$ of the BV group tested positive for HPV $D N A$ compared with $50 \%$ in the group without $B V(O R=5.0$; IC $95 \% 0.5$ a 52.9).

Conclusion: women with BV presented more Pap smear abnormalities after conization when compared to the women without $B V$, although this was not statistically significant. This association was not related to high-risk HPV DNA.

KEYWORDS: Bacterial vaginosis. Papillomavirus. Cervical intraepithelial neoplasia. Cervix: preneoplastic lesions.

\section{Referências}

1. Andersen ES, Nielsen K, Pedersen B. The reliability of preconization diagnostic evaluation in patients with cervical intraepithelial neoplasia and microinvasive carcinoma. Gynecol Oncol 1995; 59:143-7.

2. Nagai $Y$, Maehama $T$, Asato $T$, Kanazawa $K$. Persistence of human papillomavirus infection after therapeutic conization for CIN 3: is it an alarm for disease recurrence? Gynecol Oncol 2000; 79:294-9.

3. Narducci F, Occelli B, Boman F, Vinatier D, Leroy JL. Positive margins after conization and risk of persistent lesion. Gynecol Oncol 2000; 46:311-4.

4. Skejeldestat FE, Hagen B, Lie AK, Isaksen C. Residual and recurrent disease after laser conization for cervical intraepithelial neoplasia. Obstet Gynecol 1997; 90:428-33.

5. Elfgren K, Bistoletti P, Dillner L, Walboomers JM, Meijer C, Dillner J. Conization for cervical intraepithelial neoplasia is followed by disappearance of human papillomavirus deoxyribonucleide acid and decline in serum and cervical mucus antibodies against human papillomavirus antigens. Am J Obstet Gynecol 1996; 174:937-42.

6. Houfflin-Debarge V, Collinet P, Vinatier D, et al. Value of human papillomavirus testing after conization by loop electrosurgical excision for highgrade squamous intraepithelial lesions. Gynecol Oncol 2003; 90:587-92.

7. Cruickshank ME, Sharp L, Chambers G, Smart L, Murray G. Persistent infection with human papillomavirus following the successful treatment of high grade cervical intraepithelial neoplasia. BJOG 2002; 109:579-81.

8. Jain S, Tseng CJ, Horng SG, Soong YK, Pao CC. Negative predictive of human papillomavirus test following conization of the cervix uteri. Gynecol Oncol 2001; 82:177-80.

9. Kucera E, Sliutz G, Czerwenka K, Breitenecker G, Leodolter S, Reinthaller A. Is high-risk papillomavirus infection associated with intraepithelial neoplasia eliminated after conization by large-loop excision of the transformation zone? Eur J Obstet Gynecol Reprod Biol 2001; 100:72-6.

10.Lu CH, Liu FS, Tseng JJ, Ho ES. Predictive factors for residual disease in subsequent hysterectomy following conization for CIN III. Gynecol Oncol 2000; 79:284-8.

11.Kalogirou D, Antoniou G, Karakitsos P, Botsis D, Kalogirou O, Giannikos L. Predictive factors used to justify hysterectomy after loop conization: increasing age and severity of disease. Eur J Gynaecol Oncol 1997; 18:113-6.

12.Moore BC, Higgins RV, Laurent SL, Marroum MC, Bellitt P. Predictive factors from cold knife conization for residual cervical intraepithelial neoplasia in subsequent hysterectomy. Am J Obstet Gynecol 1995; 173:361-6.

13.Platz-Christensen JJ, Sundström E, Larsson PG. Bacterial vaginosis and cervical intraepithelial neoplasia. Acta Obstet Gynecol Scand 1994; 73:586-8.

14. Kharsany AB, Hoosen AA, Moodley J, Bagaratee J, Gouws E. The association between sexually transmitted pathogens and cervical intraepithelial neoplasia in a developing community. Genitourin Med 1993; 69:357-60.

15.McNicol P, Paraskevas M, Guijon F. Variability of polymerase chain reaction-based detection of human papillomavirus DNA is associated with the composition of vaginal microbial flora. J Med Virol 1994; 43:194-200.

16.Pavic N. Is there a local production of nitrosamines by the vaginal microflora in anaerobic/ trichomoniasis? Med Hypotheses 1984; 15:433-6. 
17.Behbakht K, Friedman J, Heimler I, Aroutcheva A, Simoes J, Faro S. Role of the vaginal microbiological ecosystem and cytokine profile in the promotion of cervical dysplasia: a case-control study. Infect Dis Obstet Gynecol 2002; 10:181-6.

18.Schnadig VJ, Davie KD, Shafer SK, Yandell RB, Islam MZ, Hannigan EV. The cytologist and bacterioses of the vaginal ectocervical area: clues, commas and confusion. Acta Cytol 1989; 33:287-97.

19.Figueiredo PG, Derchain SFM, Sarian LOZ, et al. Detecção de DNA de papillomavirus humano após excisão da zona de transformação com alça diatérmica para o tratamento de neoplasia intra- epitelial cervical. Rev Bras Ginecol Obstet 2003; 25:9-15.

20.Murta EDC, Souza MAH, Araujo Júnior E, Adad SJ. Incidence of Gardnerella vaginalis, Candida sp and human papillomavirus in cytological smears. São Paulo Med J 2000; 1 18:105-8.

21.Mikamo H, Sato Y, Hayasaki Y, et al. Intravaginal bacterial flora in patients with uterine cervical cancer. High incidence of detection of Gardnerella vaginalis. J Infect Chemother 1999; 5:82-5.

22.Mao C, Hughes JP, Kiviat N, et al. Clinical findings among women with human papillomavirus infection. Am J Obstet Gynecol 2003; 188:677-84.

Recebido em: 28/6/04 Aceito com modificações em: 19/10/04 